\title{
The effect of age, gender, economic state, and urbanization on the temporal trend in sudden cardiac arrest: a nationwide population-based cohort study
}

\author{
Pil-Sung Yang ${ }^{1 \dagger}$, Daehoon Kim ${ }^{2 \dagger}$, Jung-Hoon Sung ${ }^{1^{*+}}$ and Boyoung Joung ${ }^{2^{*}+}$ (1)
}

\begin{abstract}
Background: Sudden cardiac arrest (SCA) has not been well studied in Asian countries. This study investigated the temporal trends in the incidence and outcomes of SCA and the impact of age, gender, economic state, and urbanization on SCA using a nationwide population-based sample cohort of South Korea.

Methods: In the Korean National Health Insurance Service-Sample Cohort consisting of one million persons from 2003 through 2013, we identified 5,675 (0.56\%) patients with SCA using ICD-10 code 146 and I49.0. We evaluated the impact of the age, gender, household income, and urbanization level on the incidence and outcome of SCA.

Results: During the study period, the overall age- and gender-adjusted annual incidence of SCA increased by $46.9 \%$ from 30.9 in 2003 to 45.4 in 2013 (per 100,000 person-years, $p<0.001$ for trend). The medical cost per 100,000 personyears also greatly increased about four times ( $p<0.001 \mathrm{for}$ trend). The overall adjusted survival to hospital discharge rate increased from 8.9\% in 2003 to 13.2\% in 2013 (adjusted rate ratio per year 1.05; $p<0.001$ for trend). Old age and low household incomes of the population was related to increased SCA and poor survival to hospital discharge rate. The proportion of patients with intensive or advanced therapeutic modalities after SCA greatly increased from 1.6\% in 2003 to $10.0 \%$ in 2013 ( $p<0.001$ for trend). This increase was consistent regardless of age, gender, economic state, and urbanization level.
\end{abstract}

Conclusions: Although the incidence of SCA was increased, the outcome was improved for the decade. However, in the elderly and low-income population, the incidence of SCA continued to rise and survival outcome was not improved.

Keywords: Sudden cardiac arrest, Incidence, Outcome, Economic state, Aging

*Correspondence: atropin5@cha.ac.kr; cby6908@yuhs.ac

†Jung-Hoon Sung, Boyoung Joung: Joint senior authors

tPil Sung Yang a nd Daehoon Kim contributed equally to this work.

1 Department of Cardiology, CHA Bundang Medical Center, CHA

University, 59 Yatap-ro, Bundang-gu, Seongnam-si, Gyeonggi-do 13496,

Republic of Korea

${ }^{2}$ Division of Cardiology, Department of Internal Medicine, Yonsei University College of Medicine, 50-1 Yonseiro, Seodaemun-gu,

Seoul 03722, Republic of Korea

\section{Introduction}

Despite the tremendous advances in the field of cardiovascular medicine, sudden cardiac arrest (SCA) is a significant clinical and public health problem worldwide [1]. The epidemiology of SCA has been studied as an indicator of the national health. However, the estimates on the incidence and survival rate of SCA vary widely depending on the ethnicities, regions, and data sources for case ascertainment. The majority of estimates about SCA are 
based on data from the emergency medical service (EMS) for out-of-hospital SCA. In a global systemic review, the annual incidence and survival to hospital discharge rates of EMS-assessed out-of-hospital SCA were 52.5 (per 100,000 person-years) and 2\% in Asia, 86.4 (per 100,000 person-years) and 9\% in Europe, 98.1 (per 100,000 person-years) and 6\% in North America, and 112.9 (per 100,000 person-years) and 11\% in Australia, respectively [2]. In comparison to the out-of-hospital SCA, few studies have reported the incidence and outcomes of in-hospital SCA. Between one and five of every 1,000 hospital inpatients are estimated to have an in-hospital SCA in the developed countries, and less than $20 \%$ of such patients survive to discharge [3-5]. However, the epidemiology of SCA has not been well revealed in Asian countries.

South Korea is aging faster than any other country. And the lifestyle and diet of Korean people has become more westernized with the economic growth. The disease spectrum in South Korea has changed profoundly in the last few decades. Coronary artery disease (CAD) has already become the major type of heart disease and a heavy healthcare burden. Therefore, it can be expected that the incidence of SCA has been increasing in South Korea [6]. On the other hand, an improvement in the survival outcome from SCA can also be expected because of the advances in the resuscitation science, development of public education and training programs for cardiopulmonary resuscitation, and compulsory installation of automatic external defibrillators (AEDs) in public places. However, the trend of the prevalence and outcome of SCA has not been demonstrated in South Korea's general population. Only several studies about EMS-assessed out-of-hospital SCA are available [7, 8]. Therefore, we analyzed the contemporary trends in the rate of the incidence and survival to hospital discharge from SCA from 2003 to 2013 in a Korean population-based sample cohort. We also evaluated the impact of the age, gender, household income, and urbanization level on the incidence and outcome of SCA.

\section{Methods}

\section{Data source}

In South Korea, registration with the national health insurance system is compulsory for all citizens, and hence, its coverage rate is more than $99 \%$ of the country's population. Currently, the National Health Insurance Service (NHIS) manages all databases of Korea's health service utilization, including outpatient care, inpatient care, emergency medicine, dental care, and all other medically necessary services. The NHIS released a National Sample Cohort database (NHIS-NSC) for research purposes. It consists of 1,025,340 Koreans (about 2.2\% of the total population of South Korea) as an initial 2002 cohort and has followed the subjects for 12 years (2002-2013). The cohort data were sampled systematically within 1,476 strata defined by combinations of the age, gender, eligibility status, and household income. All information on the clinical visits, hospitalization, medical treatment, and prescribed drugs are included in the NHIS-NSC. The information of the subject's mortality, obtained from the Korean National Statistical Office, was also included. Disease diagnoses for all individuals were classified according to the International Classification of Diseases 10th (ICD-10) codes. The details of the NHIS-NSC are described in previous reports [9-11].

Informed consent was not specifically obtained individually because this study was based on the data from the NHIS-NSC. The data were fully anonymized and deidentified for the analysis. This study was approved by the institutional review board of Yonsei University Health System. The first author vouches for the integrity of the data and the accuracy of the results.

\section{Data collection and study population}

In the NHIS-NSC, we identified the patients with SCA using ICD-10 code I46.x (cardiac arrest) and I49.0 (ventricular fibrillation). The patients who were discharged from an emergency department or inpatient clinic with diagnosis code I46.x or I49.0 were included. The deadon-arrival subjects with an underlying cause of death reported as I46.x or I49.0 were also included. We did not use the 2002 cohort data because the initial 2002 cohort started with subjects who did not die in 2002. A total of 5,675 patients with SCA were identified between 2003 and 2013. The study population included both in-hospital and out-of-hospital SCA. The patients' age, gender, household income, and urbanization level of the region were identified. The household income levels were stratified into 10 quantiles from grade 1 to 10 , and grade 0 meant medical aid beneficiaries (low household income: grade $0-3$, middle household income: grade $4-7$, and high household income: grade 8-10). The urbanization level of the region was classified into populated urban regions (regions with a population of more than a million) and other regions. The outcome of SCA was evaluated by the rate of survival to hospital discharge.

\section{Definition and validation of SCA}

We defined the SCA by ICD-10 codes I46.x (cardiac arrest) and I49.0 (ventricular fibrillation) after excluding non-cardiac arrest. Patients diagnosed with sudden arrest accompanied by the following diagnosis were considered non-cardiac arrest; respiratory arrest (R09.0, R09.2), gastrointestinal bleeding (I85.0, K25.0, K25.4, K26.0, K26.4, K27.0, K27.4, K92.0-K92.2), brain hemorrhage (I60.x-I62.x, S06.4-S06.6), septic shock 
(A41.9, R57.2), pregnancy and delivery (O00-O99), diabetic ketoacidosis (E14.1), anaphylaxis (T78.2), and accidents including asphyxiation, drowning, poisoning, traffic accident, fall, and suicide (T71, T75.1, T36-T65, V01-V99, W00-99, X60-X84).

To evaluate the accuracy of our definition of SCA, we conducted a validation study with medical records of two independent tertiary hospitals from 2009 to 2013. We found 731 patients with code I46.x or I49.0 after excluding those with diagnosis codes for non-cardiac causes, as mentioned above. Their medical records were then reviewed by five physicians, and we ascertained the patients with true SCA. The positive predictive value was $80.2 \%$ (586 of 731 ) by using our criteria of SCA, suggesting the good diagnostic accuracy of our definition. False-positive cases were respiratory arrest (7.0\%), history of SCA (4.2\%), arrest due to cancer progression (1.9\%), accidents (1.8\%), bleeding (1.8\%), metabolic acidosis (1.0\%), septic shock $(0.8 \%)$, stroke $(0.5 \%)$, and others $(0.9 \%)$.

\section{Statistical analysis}

To evaluate the changes in the variables by the calendar year, we used the Cochran-Armitage trend test for categorical variables and linear regression for continuous variables. The age and gender standardized incidence rates per 100,000 person-years for the study population were calculated to compare the trends by years and strata with the year 2003 as the reference (direct standardization method).

To assess whether survival to hospital discharge had changed over time, multivariable Poisson regression models were constructed. The adjusted variables in the model were the age, gender, household income, and urbanization level of the region. The Poisson distribution was used to directly estimate the rate ratios instead of the odd ratios to avoid any potential exaggeration [12]. Our independent variable, the calendar year, was included as a categorical variable, with 2003 as the reference year. We multiplied the adjusted rate ratio for each year (2004 through 2013) by the observed survival rate for the reference year to obtain the yearly risk-adjusted survival rates for the study period. Those rates represented the estimated survival for each year if the patient case mix was identical to that in the reference year [5]. We also evaluated the calendar year as a continuous variable to obtain the adjusted rate ratios for the year-to-year survival trends.

All statistical analyses were conducted with the use of SPSS 20, R 3.2.1, or GraphPad Prism 6 software. All hypothesis tests were two-sided, with a significance level of 0.05 .

\section{Results}

Patient characteristics, incidences, and medical care cost

From 2003 to 2013, a total of 5,675 patients with SCA were identified in the NHIS-NSC. Table 1 shows the temporal trends in the number and characteristics of the patients with SCA over 11 years. The absolute number of patients with SCA increased from 314 in 2003 to 677 in $2013(p<0.001$ for trend). The mean age of the SCA patients increased from $64.6 \pm 15.9$ in 2003 to $68.1 \pm 16.3$ in 2013 ( $p=0.003$ for trend), and consequently, the proportion of the elderly ( $\geq 60$ years old) in the SCA patients gradually increased ( $p=0.027$ for trend). SCA occurred more frequently in men $(59.3 \%)$ than women $(40.7 \%)$ $(p<0.001)$. However, the ratio of the genders did not significantly change during 11 years. The proportion of patients with SCA in the middle- and high-income brackets decreased $(p<0.001$ and $p=0.002$ for trend, respectively), and that in the low-income bracket increased $(p<0.001$ for trend).

Figure 1 shows the temporal trends in the annual incidence and medical care cost of SCA per 100,000 personsyears. The crude annual incidence rate of SCA without an adjustment for the age and gender increased from 30.9 in 2003 to 66.7 in 2013 (per 100,000 person-years, $p<0.001$ for trend). After adjusting for the age and gender, the annual incidence of SCA increased by about 1.5 times from 30.9 in 2003 to 45.4 in 2013 (per 100,000 personyears, $p<0.001$ for trend). Along with the increasing incidence of SCA, the medical care cost for SCA per 100,000 person-years also greatly increased from $\$ 65,866$ in 2003 to $\$ 241,534$ even after an adjustment for the inflation rate in South Korea during the 11 years ( $p<0.001$ for trend).

\section{The effect of the age, gender, household income, and urbanization level of the region on the incidence}

The age, gender-adjusted incidence rate of SCA according to the age, gender, household income, and urbanization level of the region are presented in Fig. 2. The increase of SCA was particularly predominant in the elderly $\geq 60$ years of age (from 160.0 in 2003 to 238.9 in 2013, per 100,000 person-years, $p<0.001$ for trend) (Fig. 2a). There were no temporal differences in the patients' gender during the study period (all $p<0.001$ for trend, the ratio of two groups: $p=0.209$ for trend) (Fig. 2b). The incidence of SCA per 100,000 person-years increased significantly in the subjects with a low economic status (from 31.5 in 2003 to 65.9 in 2013, $p<0.001$ for trend), and a statistically significant difference between the low and middle-high household income groups has arisen after 2011 (Fig. 2c). There was no temporal difference in the proportion of patients with SCA according to the urbanization level of the region during 


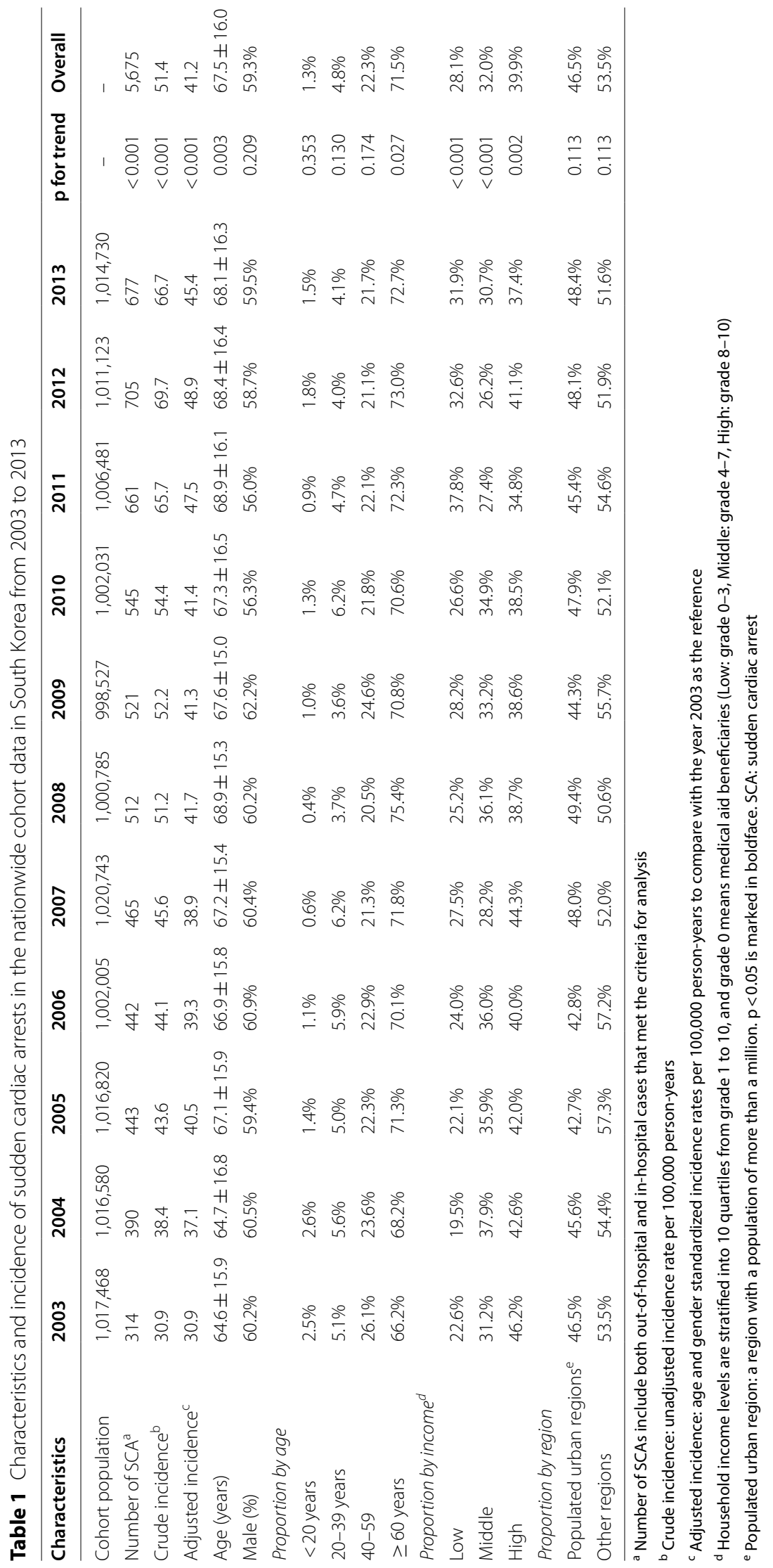




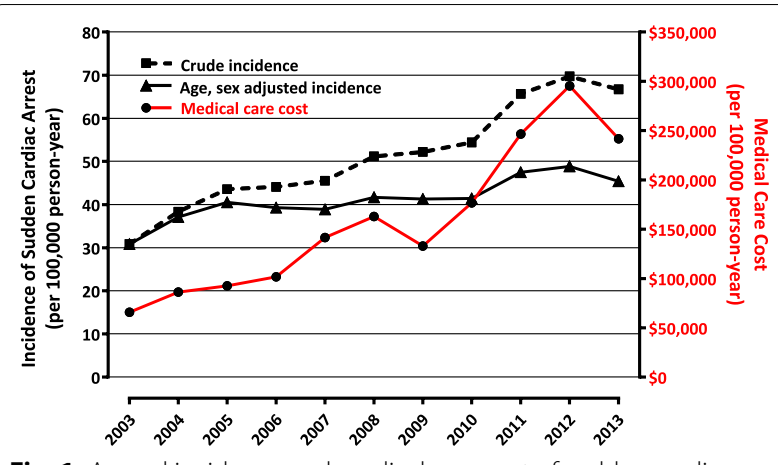

Fig. 1 Annual incidence and medical care cost of sudden cardiac arrests per 100,000 persons in South Korea from 2003 to 2013. The medical care cost was adjusted for the inflation rate in South Korea. $p<0.001$ for trends for all variables the study period (all $p<0.001$ for trend, the ratio of two groups: $p=0.113$ for trend) (Fig. $2 \mathrm{~d}$ ).

\section{Survival to hospital discharge}

During the study interval of 2003 to 2013, the overall rate of survival to discharge was $8.6 \%$ (487 of 5,675 patients). There was a significant trend toward an increased survival to hospital discharge for all study patients from $8.9 \%$ in 2003 to $11.2 \%$ in 2013 ( $\mathrm{p}=0.006$ for trend) (Fig. 3a). In a subgroup analysis, the survival to hospital discharge rates significantly improved in the subjects younger than 60 years ( $p<0.001$ for trend) (Fig. 3b), males $(p<0.001$ for trend) (Fig. 3c), and the middle-high household income groups ( $p=0.001$ for trend) (Fig. 3d). However, the trend toward an increased survival was not observed in the patients older than 60 years $(p=0.492$ for trend) (Fig. 3b), females ( $p=0.960$ for trend) (Fig. 3c), and those with a low household income $(p=0.120$ for trend) (Fig. 3d).
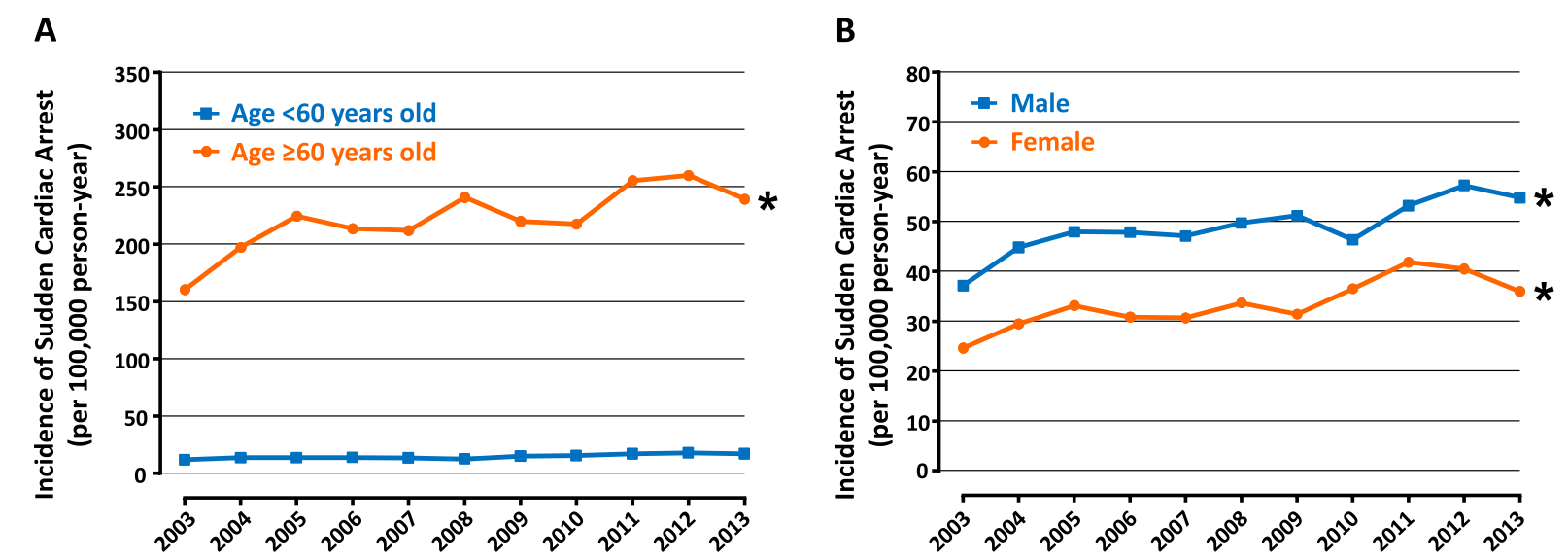

C

D
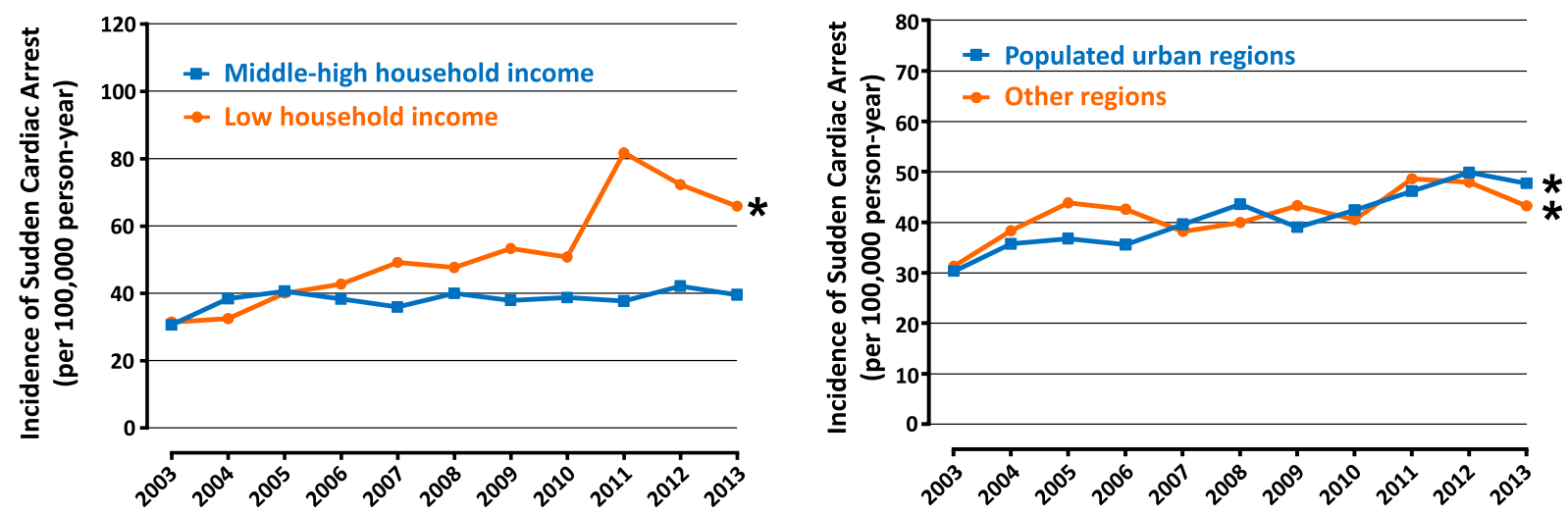

Fig. 2 Annual incidence of sudden cardiac arrest per 100,000 persons according to the age (a), Gender (b), Household income (c), and Region (d) from 2003 to 2013. All annual incidences were adjusted for the age and gender. ${ }^{*} p \leq 0.001$ for trend 


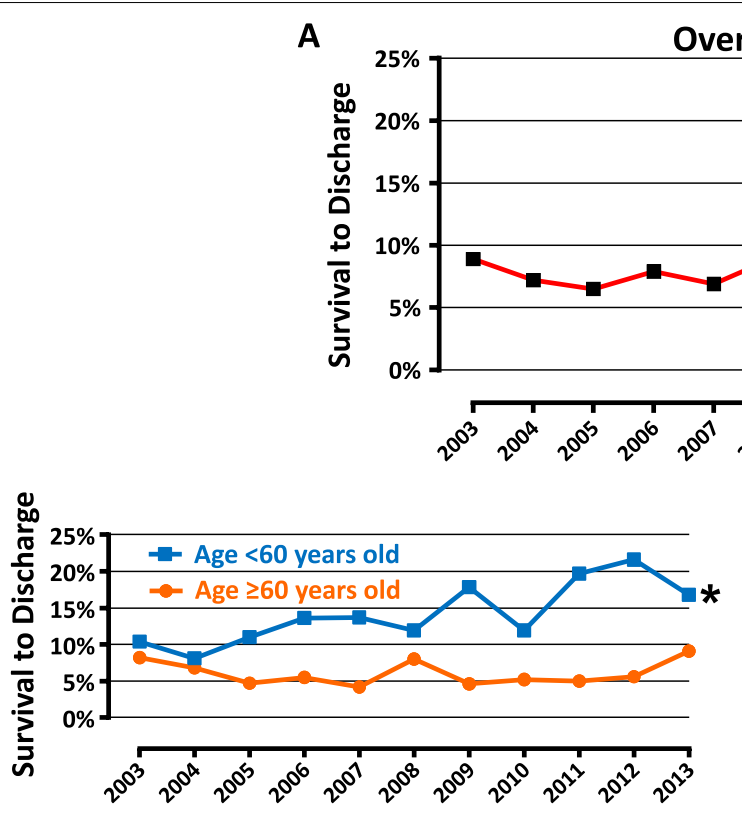

B

D

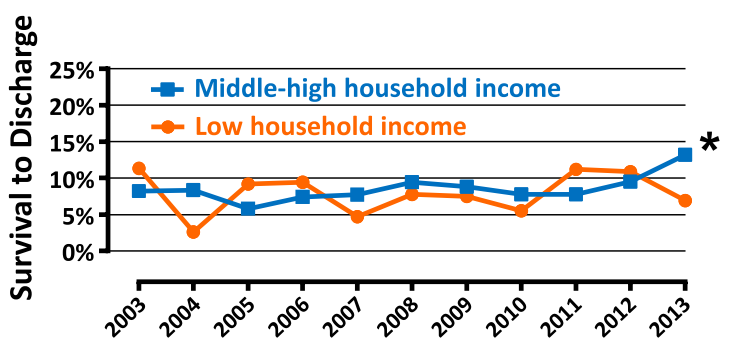

C

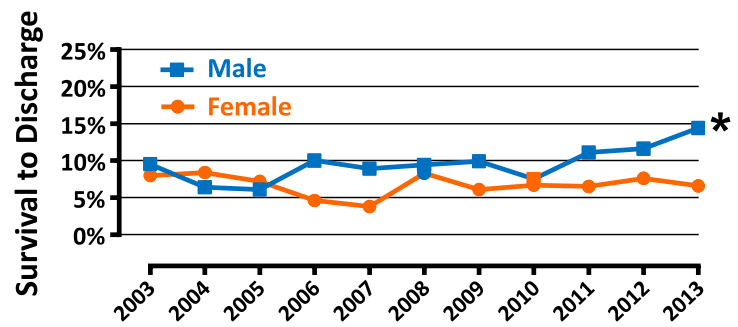

E

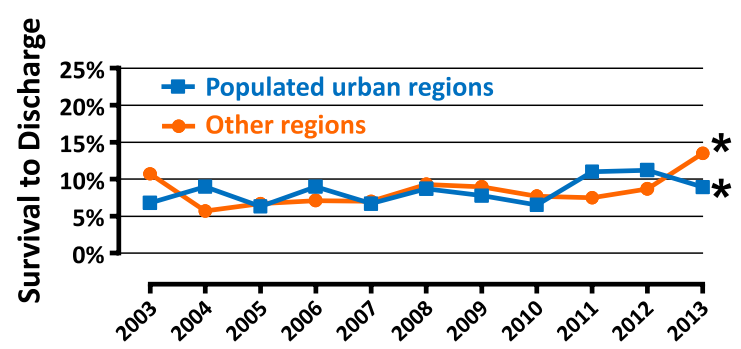

Fig. 3 Survival rate to hospital discharge of sudden cardiac arrests from 2003 to 2013, including the total (a) and According to the age (b), Gender (c), Household income (d), and Region by urbanization (e). ${ }^{*} p \leq 0.001$ for trend

There was no difference in the survival improvement between the populated urban, and other regions (all $p<0.001$ for trend, the ratio of two groups: $p=0.544$ for trend) (Fig. 3e).

After an adjustment for the temporal trends in the patient characteristic such as the age, gender, household income, and regions, the overall survival improved from $8.9 \%$ in 2003 to $13.2 \%$ in 2013 (adjusted rate ratio per year, 1.05; 95\% confidence interval [CI], 1.02 to 1.08; $\mathrm{p}$ for trend $<0.001$, Table 2).

\section{Trends in the treatment modality for SCA}

The temporal trends in the treatment modality for SCA are presented in Fig. 4. The proportion of patients with any intensive or advanced therapeutic modalities after SCA greatly increased from $1.6 \%$ in 2003 to $10.0 \%$ in 2013 ( $p<0.001$ for trend) (Fig. 4a). The proportion of patients who underwent percutaneous transluminal coronary angioplasty (PTCA) during the hospitalization for SCA increased from $0.68 \%$ in 2003 to $2.68 \%$ in $2013(p<0.001$ for trend). The proportion of patients who underwent continuous renal replacement therapy (CRRT) also increased from $1.02 \%$ in 2003 to $5.52 \%$ in $2013(p<0.001$ for trend). Insurance claims for implantable cardioverter defibrillators (ICDs) and therapeutic hypothermia were first recorded in 2008 and 2010, respectively. ICD implantations after SCA increased from $0.21 \%$ of cases in 2008 to $0.79 \%$ of cases in 2013 ( $p<0.001$ for trend). Therapeutic hypothermia for SCA increased from $0.4 \%$ of cases in 2010 to $3.0 \%$ of cases in $2013(p<0.001$ for trend).

This proportion of patients with any intensive or advanced therapeutic modalities after SCA increased regardless of age, gender, household income and urbanization level (Fig. 4b-e).

\section{Discussion}

The main findings are as follows. First, the annual incidence of SCA in South Korea increased over the past decade and that increase was particularly predominant in the elderly ( $\geq 60$ years old) and those with a low economic status. Second, the survival outcome from SCA improved 
Table 2 Model-adjusted trends in the rates of survival to hospital discharge

\begin{tabular}{llll}
\hline Year & $\begin{array}{l}\text { Unadjusted } \\
\text { rate } \\
(\%)\end{array}$ & $\begin{array}{c}\text { aAdjusted rate } \\
\text { Adjusted rate } \\
\text { ratio (95\% Cl) }\end{array}$ \\
\hline 2003 & 8.9 & reference & reference \\
2004 & 7.2 & 7.3 & $0.82(0.49-1.34)$ \\
2005 & 6.5 & 7.1 & $0.80(0.49-1.30)$ \\
2006 & 7.9 & 8.7 & $0.98(0.62-1.56)$ \\
2007 & 6.9 & 7.9 & $0.89(0.56-1.43)$ \\
2008 & 9.0 & 11.1 & $1.25(0.80-1.95)$ \\
2009 & 8.4 & 9.8 & $1.10(0.71-1.70)$ \\
2010 & 7.2 & 8.2 & $0.92(0.58-1.44)$ \\
2011 & 9.1 & 11.1 & $1.25(0.83-1.91)$ \\
2012 & 9.9 & 11.6 & $1.30(0.87-1.96)$ \\
2013 & 11.2 & 13.2 & $1.48(1.00-2.23)$ \\
${ }^{b}$ Adjusted rate ratio per year (95\% Cl) & & $1.05(1.02-1.08)$ \\
${ }^{b}$ p vale for trend & & & $<0.001$ \\
\hline
\end{tabular}

The model was adjusted for the age, gender, household income, and regions. There were no interactions

${ }^{a}$ Model-adjusted rates of survival to discharge for each year were obtained by multiplying the observed rate for the reference year (2003) by the corresponding rate ratios from a model evaluating the year as a categorical variable

${ }^{b}$ Adjusted rate ratio per year and $p$-value for trend were calculated with a model evaluating the year as a continuous variable. Cl: confidence interval

overall, particularly in the young and middle-aged populations, but not in the elderly patients ( $\geq 60$ years old), females, and those with a low household income. Finally, intensive or advanced modalities were increasingly used for the treatment of SCA.

\section{Increasing incidence of SCA}

In Western countries, the incidence of SCA has declined, in parallel with the decline in CAD mortality $[13,14]$. In contrast, the incidence of SCA in South Korea has increased over the past decade. Lifestyle changes with economic growth and a rapidly aging population might be the main cause of the increased incidence of SCA in South Korea. Although the incidence of CAD in South Korea is lower than that in Western countries [15], CAD has increased in recent years [6] due to changes in lifestyle. In addition, the overall incidence of SCA is higher among older patients [16]. Consistently, this study showed that the increase in SCA was particularly predominant in patients $\geq 60$ years of age.

The incidence of SCA was higher in the lower-income rather than higher-income groups in the United States and Canada $[17,18]$. In South Korea, the age and gender standardized incidence rate of SCA was also higher in the low household income group rather than the middle-high household income group. Looking more closely, there was no statistically significant difference in the incidence of SCA between the low and middle-high income groups from 2003 to 2010. However, a significant difference in the incidence of SCA between the low and middle-high income groups was observed after 2011 because of the marked increase in SCA in patients with a low economic status. This finding suggests that the social imbalances are deepening in South Korea. A lower economic status has been associated with an increased burden of risk factors [19], and a decreased likelihood of receiving proper risk management or treatment for cardiovascular disease [20]. Thus, the population-based interventions from the National Health Insurance Database Cohort in South Korea to reduce the cardiovascular risk factors and treat unrecognized cardiovascular disease in low-income communities may prove to be effective in reducing the disparities in the incidence of SCA.

\section{Improved outcome of SCA}

Several studies from Western countries have reported improved survival rates of SCA during the past decade $[5,21]$. Survival from SCA in South Korea also improved between 2003 and 2013. In the past ten years, many advances in resuscitation science such as therapeutic hypothermia and AEDs have been introduced into South Korea. We showed that the utilization of intensive or advanced modalities for the treatment of SCA such as PTCA, ICDs, CRRT, and therapeutic hypothermia have increased. Moreover, government activities and laws to develop public education and training programs for cardiopulmonary resuscitation were enacted in 2002, and the actual training program began later in 2006. AEDs in public places became compulsory in 2008 and were expanded to more private places in 2012. These changes might have been what made the improvement in the survival from SCA in South Korea during the past ten years.

In this study, the overall rate of survival to hospital discharge after SCA in South Korea was $8.6 \%$. Although it is comparable to that reported by studies conducted in North America (7.9-11.4\%), Europe (10.7\%), and Japan (12\%) during the same time [22-25], the outcome of SCA in South Korea is still relatively backward compared to the other developed countries. Moreover, a disparity in the outcome of SCA among the social stratum existed. The improvement in the SCA outcome in the overall population was attributable to the increased survival rate in the young and middle-aged populations $(<60$ years old), a male gender, and those with middle-high household incomes. The outcomes of SCA in the elderly ( $\geq 60$ years old), females, and those with a low household income did not improve. The social and medical systems in South Korea for SCA are still less than optimal compared to Western developed countries, and we need to make more effort. 


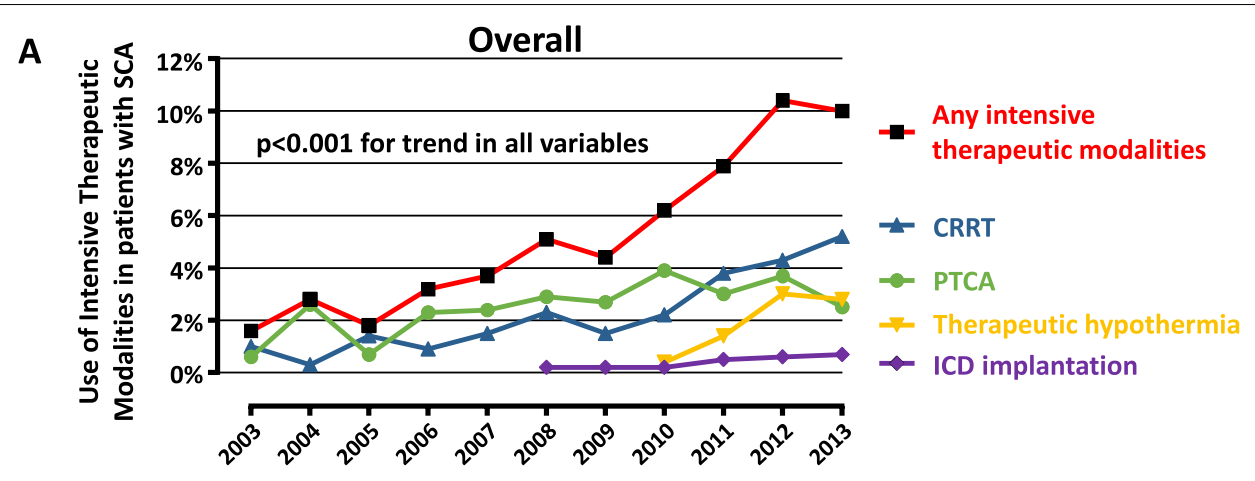

B

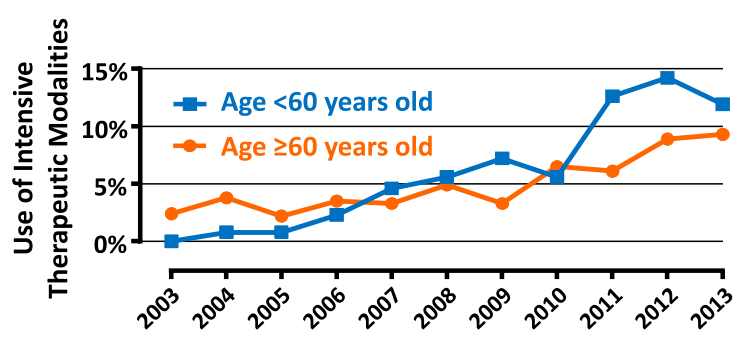

D

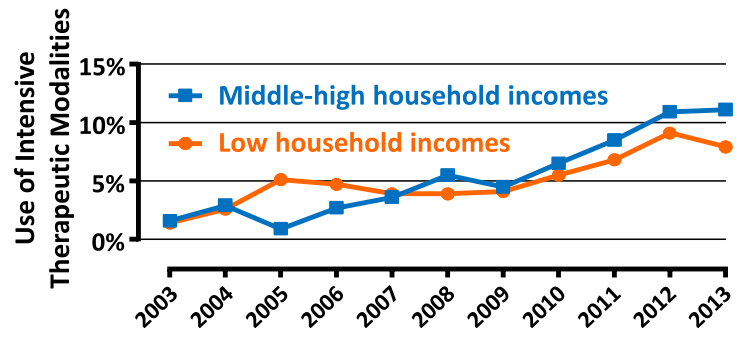

C

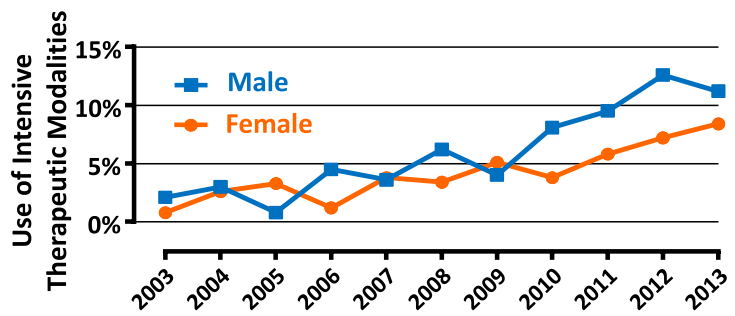

E

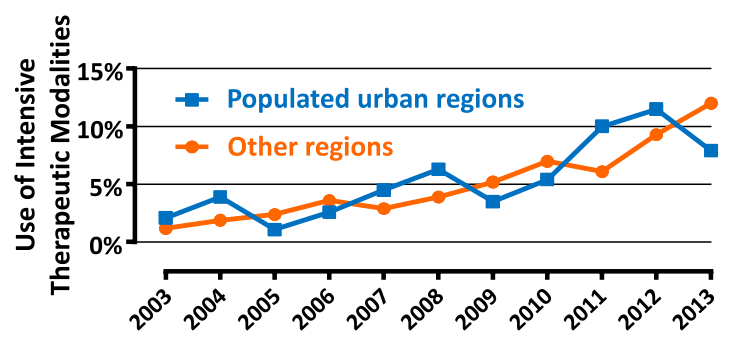

Fig. 4 a Annual proportion of patients who underwent PTCA, CRRT, therapeutic hypothermia, and ICD implantation after sudden cardiac arrests in South Korea from 2003 to 2013. b-e The proportion of patients who underwent any intensive therapeutic modalities according to the age (b), Gender (c), Household income (d), and Region by urbanization (e) The data are based on the medical insurance claims of each treatment. Any intensive therapeutic modalities: PTCA CRRT, therapeutic hypothermia, or ICD implantation; CRRT continuous renal replacement therapy ICD implantable cardioverter defibrillator; PTCA percutaneous transluminal coronary angioplasty. $p<0.001$ for trends in all variables

There is no significant difference in intensive therapeutic modalities according to the household income. The reason why the proportion of SCA patients who receive intensive therapeutic modalities does not differ significantly depending on household income may be because South Korea has a well-established national health insurance system covering the entire citizens. Nevertheless, the survival rate of the low household income group was relatively low, there was no improvement compared to the middle-high household income group. It can be explained by several reasons. First, it is well known that a lower economic status has been associated with an increased burden of cardiovascular risk factors. Higher rates of cardiovascular risk factors in the low-income group will affect not only the higher incidence rate of SCA but also the lower survival rate after SCA compared to the high-income group. Second, the subjects in the low-income group are more likely to live alone and are more likely to be excluded from the daily care. This reduces the chances of being discovered by a witness in an SCA, receiving bystander CPR, and surviving transport to the hospital. To examine the relationship between income level and the likelihood that a patient with SCA would be discovered by a witness, we identified patients whose cause of death was listed as SCA on the death certificate and had no hospital records. They died without being found alive and were unable to visit the hospital. The proportion of these patients was higher with lower income $(9.0 \%, 6.3 \%$, and $4.9 \%$ in the low, middle, and high household income groups, respectively).

In our analysis, survival after SCA improved gradually over 10 years in men but not in women. Among the elderly living alone who have a high risk of death in the event of SCA, the proportion of women was more than 
twice that of men in South Korea (From Korean Statistical Information Service; https://kosis.kr). The average number of diseases among elderly females living alone was 1.6 times higher than that of men. Nearly half of the elderly females living alone also lived in isolation from social networks. These social differences between men and women may be one of the reasons why survival rates after SCA did not improve in female patients.

\section{Data for SCA in South Korea}

Several studies about EMS-assessed out-of-hospital SCAs in South Korea have been published [7, 8]. However, those data could be an underestimate, because out-of-hospital SCAs not attended by emergency medical personnel were not included. Moreover, there is little data for in-hospital SCAs in South Korea. Understanding the accurate characteristics and temporal trends of SCAs in the community are critical for developing appropriate interventions. Because the nationwide cohort such as the NHIS-NSC used in this study would enable a more accurate determination of the overall SCA burden in the community including both out-of-hospital and in-hospital cases, it can complement the EMS-based registry data on SCAs.

\section{Study limitations}

This study has several limitations. First, all patient data were from the insurance claim data provided by the NHIS-NSC. The precision of the SCA diagnosis, which was based on ICD-10 code from the administrative data reported by physicians, may be a concern. Potential misclassifications might have existed. Second, the site of the SCA including the in-hospital and out-of-hospital settings and initial rhythm status of the patients could not be classified in the NHIS-NSC database despite those factors being closely associated with the outcome of SCA.

\section{Conclusions}

The incidence of SCA in South Korea continues to have risen over the decade, especially in the elderly and lowincome bracket. The survival after SCA has improved significantly with the increase in the utilization of intensive or advanced modalities. However, survival improvement was not observed in the elderly patients, females, and those with a low household income.

\section{Abbreviations}

AED: Automatic external defibrillator; CRRT: Continuous renal replacement therapy; CAD: Coronary artery disease; EMS: Emergency medical service; ICD: Implantable cardioverter defibrillator; ICD-10: International classification of diseases 10th; NHIS: National health insurance service; NHIS-NSC: National health insurance service-National sample cohort; PTCA: Percutaneous transluminal coronary angioplasty; SCA: Sudden cardiac arrest.

\section{Acknowledgments}

The NHIS of Korea provided the National Health Information Database. The authors would like to thank the National Health Insurance Service for their cooperation.

\section{Authors' contributions}

BJ and J-HS contributed to the conception and design of the work and critical revision of the manuscript. P-SY and DK contributed to the conception and design of the work, interpretation of data for the work, and drafting of the manuscript.

\section{Funding}

This study was supported by a research grant from the Korean Healthcare Technology R\&D project funded by the Ministry of Health \& Welfare (HI15C1200, HC19C0130). Ministry of health and welfare, HI15C1200, Boyoung Joung, HC19C0130, Boyoung Joung

\section{Availability of supporting data}

The data underlying this article are available in the National Health Insurance Sharing Service (NHISS) of Korea at https://nhiss.nhis.or.kr. Applications to use the data will be reviewed by the inquiry committee of research support and, once approved, raw data will be provided to the authorized researcher with a fee at several permitted sites.

\section{Declarations}

Ethics approval and consent to participate

This study was approved by the Institutional Review Board of the Yonsei University Health System (4-2016-0329). The requirement for informed consent was waived because personal identification information was removed after cohort generation, in accordance with the strict confidentiality guidelines.

\section{Competing interests}

Dr. Boyoung Joung has served as a speaker for Bayer, BMS/Pfizer, Medtronic, and Daiichi-Sankyo and received research funds from Medtronic and Abbott. Neither author has received any fees directly. The other authors have nothing to declare.

\section{Consent for publication}

All authors have permitted the publication.

Received: 15 June 2021 Accepted: 10 December 2021

Published online: 08 March 2022

\section{References}

1. Hayashi M, Shimizu W, Albert CM. The spectrum of epidemiology underlying sudden cardiac death. Circ Res. 2015;1 16:1887-906. https:// doi.org/10.1161/CIRCRESAHA.116.304521.

2. Berdowski J, Berg RA, Tijssen JG, Koster RW. Global incidences of outof-hospital cardiac arrest and survival rates: Systematic review of 67 prospective studies. Resuscitation. 2010;81:1479-87. https://doi.org/10. 1016/j.resuscitation.2010.08.006.

3. Sandroni C, Nolan J, Cavallaro F, Antonelli M. In-hospital cardiac arrest: incidence, prognosis and possible measures to improve survival. Intensive Care Med. 2007;33:237-45. https://doi.org/10.1007/ s00134-006-0326-z.

4. Merchant RM, Yang L, Becker LB, Berg RA, Nadkarni V, Nichol G, Carr BG, Mitra N, Bradley SM, Abella BS, American Heart Association Get With The Guidelines-Resuscitation I. Groeneveld PW 2011 Incidence of treated cardiac arrest in hospitalized patients in the United States. Crit Care Med. 2011;39:2401-6. https://doi.org/10.1097/CCM.0b013e3182 257459.

5. Girotra S, Nallamothu BK, Spertus JA, Li Y, Krumholz HM, Chan PS, Trends in survival after in-hospital cardiac arrest. Trends in survival after 
in-hospital cardiac arrest. N Engl J Med. 2012;367:1912-20. https://doi. org/10.1056/NEJMoa1109148.

6. Hong JS, Kang HC, Lee SH, Kim J. Long-term trend in the incidence of acute myocardial infarction in Korea: 1997-2007. Korean Circ J. 2009:39:467-76. https://doi.org/10.4070/kcj.2009.39.11.467.

7. Ro YS, Shin SD, Kitamura T, Lee EJ, Kajino K, Song KJ, Nishiyama C, Kong SY, Sakai T, Nishiuchi T, Hayashi Y, Iwami T, Seoul-Osaka Resuscitation Study G. Temporal trends in out-of-hospital cardiac arrest survival outcomes between two metropolitan communities: Seoul-Osaka resuscitation study. BMJ Open. 2015;5:007626. https://doi.org/10.1136/bmjop en-2015-007626.

8. Yang HJ, Kim GW, Kim H, Cho JS, Rho TH, Yoon HD, Lee MJ, Consortium $\mathrm{N}-\mathrm{C}$. Epidemiology and outcomes in out-of-hospital cardiac arrest: a report from the NEDIS-based cardiac arrest registry in Korea. J Korean Med Sci. 2015;30:95-103. https://doi.org/10.3346/jkms.2015.30.1.95.

9. Lee J, Lee JS, Park SH, Shin SA, Kim K. Cohort profile: the national health insurance service-national sample cohort (NHIS-NSC). South Korea Int J Epidemiol. 2016. https://doi.org/10.1093/ije/dyv319.

10. Kim NH, Lee J, Kim TJ, Kim NH, Choi KM, Baik SH, Choi DS, Pop-Busui R, Park Y, Kim SG. Body mass index and mortality in the general population and in subjects with chronic disease in korea: a nationwide cohort study (2002-2010). PLoS ONE. 2015;10:e0139924. https://doi.org/10.1371/journ al.pone.0139924.

11. Han KT, Jang SY, Park S, Cho KH, Yoo KB, Choi Y, Park EC. Social welfare centers protect outpatients with mood disorders from risk of hospital admission. PLoS ONE. 2016;11:e0146754. https://doi.org/10.1371/journal. pone.0146754.

12. Zou G. A modified poisson regression approach to prospective studies with binary data. Am J Epidemiol. 2004;159:702-6.

13. Ni H, Coady S, Rosamond W, Folsom AR, Chambless L, Russell SD, Sorlie PD. Trends from 1987 to 2004 in sudden death due to coronary heart disease: the atherosclerosis risk in communities (ARIC) study. Am Heart J. 2009;157:46-52. https://doi.org/10.1016/j.ahj.2008.08.016.

14. Niemeijer MN, van den Berg ME, Leening MJ, Hofman A, Franco OH, Deckers JW, Heeringa J, Rijnbeek PR, Stricker BH, Eijgelsheim M. Declining incidence of sudden cardiac death from 1990-2010 in a general middleaged and elderly population: the Rotterdam Study. Heart Rhythm. 2015;12:123-9. https://doi.org/10.1016/j.hrthm.2014.09.054.

15. Ueshima H, Sekikawa A, Miura K, Turin TC, Takashima N, Kita Y, Watanabe M, Kadota A, Okuda N, Kadowaki T, Nakamura Y, Okamura T. Cardiovascular disease and risk factors in Asia: a selected review. Circulation. 2008:118:2702-9. https://doi.org/10.1161/CIRCULATIONAHA.108.790048.

16. Chugh SS, Jui J, Gunson K, Stecker EC, John BT, Thompson B, llias N, Vickers C, Dogra V, Daya M, Kron J, Zheng ZJ, Mensah G, McAnulty J. Current burden of sudden cardiac death: multiple source surveillance versus retrospective death certificate-based review in a large U.S. community. J Am Coll Cardiol. 2004;44:1268-75. https://doi.org/10.1016/j.jacc.2004.06. 029.

17. Reinier K, Stecker EC, Vickers C, Gunson K, Jui J, Chugh SS. Incidence of sudden cardiac arrest is higher in areas of low socioeconomic status: a prospective two year study in a large United States community. Resuscitation. 2006;70:186-92. https://doi.org/10.1016/j.resuscitation.2005.11. 018.

18. Reinier K, Thomas E, Andrusiek DL, Aufderheide TP, Brooks SC, Callaway CW, Pepe PE, Rea TD, Schmicker RH, Vaillancourt C, Chugh SS, Resuscitation Outcomes Consortium I. Socioeconomic status and incidence of sudden cardiac arrest. CMAJ. 2011;183:1705-12. https://doi.org/10.1503/ cmaj.101512.

19. Winkleby MA, Jatulis DE, Frank E, Fortmann SP. Socioeconomic status and health: how education, income, and occupation contribute to risk factors for cardiovascular disease. Am J Public Health. 1992;82:816-20.

20. McWilliams JM, Meara E, Zaslavsky AM, Ayanian JZ. Differences in control of cardiovascular disease and diabetes by race, ethnicity, and education: U.S. trends from 1999 to 2006 and effects of medicare coverage. Ann Intern Med. 2009;150:505-15.

21. Chan PS, McNally B, Tang F, Kellermann A, Group CS. Recent trends in survival from out-of-hospital cardiac arrest in the United States. Circulation. 2014;130:1876-82. https://doi.org/10.1161/CIRCULATIONAHA.114. 009711.

22. Nichol G, Thomas E, Callaway CW, Hedges J, Powell JL, Aufderheide TP, Rea T, Lowe R, Brown T, Dreyer J, Davis D, Idris A. Stiell I and Resuscitation
Outcomes Consortium I. Regional variation in out-of-hospital cardiac arrest incidence and outcome. JAMA. 2008;300:1423-31. https://doi.org/ 10.1001/jama.300.12.1423.

23. Brooks SC, Schmicker RH, Rea TD, Aufderheide TP, Davis DP, Morrison LJ, Sahni R, Sears GK, Griffiths DE, Sopko G, Emerson SS, Dorian P, Investigators ROC. Out-of-hospital cardiac arrest frequency and survival: evidence for temporal variability. Resuscitation. 2010;81:175-81. https://doi.org/10. 1016/j.resuscitation.2009.10.021.

24. Atwood C, Eisenberg MS, Herlitz J, Rea TD. Incidence of EMS-treated outof-hospital cardiac arrest in Europe. Resuscitation. 2005;67:75-80. https:// doi.org/10.1016/j.resuscitation.2005.03.021.

25. Iwami T, Nichol G, Hiraide A, Hayashi Y, Nishiuchi T, Kajino K, Morita H, Yukioka H, Ikeuchi H, Sugimoto H, Nonogi H, Kawamura T. Continuous improvements in "chain of survival" increased survival after out-ofhospital cardiac arrests: a large-scale population-based study. Circulation. 2009;119:728-34. https://doi.org/10.1161/CIRCULATIONAHA.108.802058.

Ready to submit your research? Choose BMC and benefit from

- fast, convenient online submission

- thorough peer review by experienced researchers in your field

- rapid publication on acceptance

- support for research data, including large and complex data types

- gold Open Access which fosters wider collaboration and increased citations

- maximum visibility for your research: over 100M website views per year

At BMC, research is always in progress.

Learn more biomedcentral.com/submissions 